Check for updates

Cite this: RSC Adv., 2019, 9, 36040

\title{
Enhanced osteogenesis of mesenchymal stem cells on electrospun cellulose nanocrystals/poly $(\varepsilon-$ caprolactone) nanofibers on graphene oxide substrates $\dagger$
}

\begin{abstract}
Dinesh K. Patel, $\hat{t}^{\mathrm{a}}$ Yu-Ri Seo, $\hbar^{\mathrm{b}}$ Sayan Deb Dutta ${ }^{\mathrm{b}}$ and Ki-Taek Lim (D) *b
Cellulose nanocrystals (CNCs) have received a great amount of attention to the production of micro/nanoplatforms for tissue engineering applications. CNCs were extracted from rice husk biomass and characterized by different spectroscopic techniques. The biocompatibility of the extracted CNCs was revealed by the WST-1 assay technique in the presence of human mesenchymal stem cells (hMSCs) after different time intervals. An improvement in the mechanical properties was observed in the fabricated scaffolds (PCL/CNC) compared to PCL scaffolds. Graphene oxide (GO)-coated (PCL/CNC) electrospun scaffolds (GPC) were prepared by the deposition of PCL/CNC composite nanofibers on the surface of GO for tissue engineering. Notably, better cell proliferation and differentiation were observed in the presence of the fabricated scaffolds. This enhancement of the properties of the fabricated scaffolds was due to the presence of conductive GO moieties which facilitated the cellular response. Therefore, the fabricated materials have the potential to be used as a biomaterial for enhanced cell proliferation and osteogenic differentiation.
\end{abstract}

Received 12th August 2019
Accepted 27th October 2019

DOI: $10.1039 / c 9 r a 06260 b$

rsc.li/rsc-advances

\section{Introduction}

Different materials have been extensively applied to produce a suitable platform as an extracellular matrix (ECM) for tissue engineering applications. ${ }^{1}$ The scaffolds' design and construction are essential for tissue engineering and regenerative medicine areas. There are some specific criteria to act as an ECM for tissue engineering, and these are: (i) it is designed as a three-dimension porous structure to promote nutrients and metabolism, (ii) it should be biodegradable and absorbed similar to the rate of the tissue regeneration, (iii) it has the appropriate mechanical properties required for the implant site, and (iv) it has excellent biocompatibility in vivo. ${ }^{2}$ The electrospinning technique is commonly used for the fabrication of high-quality scaffolds for various applications. ${ }^{3}$ The diameter of produced fibers is between a few nanometers to microns $(>1$ $\mu \mathrm{m}) .^{4-6}$ Poly- $\varepsilon$-caprolactone (PCL), a kind of biodegradable polyester polymer is commonly utilized for the fabrication of scaffolds due to its excellent physiochemical properties.?

${ }^{a}$ The Institute of Forest Science, Kangwon National University, Chuncheon 24341, Republic of Korea

${ }^{b}$ Department of Biosystems Engineering, College of Agriculture and Life Sciences, Kangwon National University, Chuncheon 24341, Republic of Korea.E-mail: ktlim@ kangwon.ac. $k r$

$\dagger$ Electronic supplementary information (ESI) available. See DOI: 10.1039/c9ra06260b

\$ Equivalent authors.
However, the hydrophobicity of PCL restricts its use as an ideal material for scaffold fabrication for massive applications. ${ }^{8}$ This limitation can be minimized by using a suitable filler in the polymer matrix. Different fillers such as nanocellulose, clay, and layered double hydroxide (LDH) are frequently used for improving the properties of the polymer. ${ }^{9}$ Among them, nanocellulose has received an enormous amount of attention from the research community due to its superior physiochemical properties. ${ }^{10}$ Cellulose nanocrystal (CNC) is a possible approach to improve the mechanical resilience of the scaffolds and to overcome the disadvantages of PCL without compromising the biological performances. ${ }^{11,12}$ It has been noted that CNCs-based polymer composites have wide potentials and can be employed in different fields..$^{13}$ In particular, a series of glycerol-plasticized starch composites reinforced by rice-husk CNCs was proven that CNCs exhibited a higher reinforcing efficiency in the plasticized starch biocomposites than at any other filler loading. ${ }^{14}$ Polymer/CNCs composites have been studied from the last several decades in tissue engineering applications. ${ }^{15-24}$ Besides, it has been found that the external stimuli including the electrical field have wide effects on cell proliferation in the presence of CNCs. ${ }^{25}$ It was observed that CNCs have low electroactive materials, and this property can be tuned by using highly conductive materials such as graphene or carbon nanotube with CNCs. ${ }^{14}$ Recent studies have reported that currentinduced graphene substrates have enhanced cell viability and osteogenesis. ${ }^{26}$ The conducting materials facilitate cellular 
response and hydroxyapatite forming ability with physioelectrical signal transfer and consequently enhanced osteogenesis. ${ }^{27,28}$ An enhancement in the bone tissues formation was noted in the presence of electrically conductive borate-based bioactive glass scaffolds by Turk et al. ${ }^{28}$ Graphene, a single carbon atom $\mathrm{sp}^{2}$ hybridized with honeycomb lattice structure has obtained a significant amount of interest from the scientific community to use as a filler material owing to their excellent physiochemical properties. ${ }^{29}$

In this study, we extracted CNCs from rice husk biomass and exploited as a filler for the fabrication of PCL/CNCs (PC) electrospun scaffolds for tissue engineering. Significant enhancement in the mechanical strength was observed in the composite electrospun than the pure polymer scaffold. Notably, the better cellular response was also noted in the composite scaffold microenvironments. Additionally, the coating of graphene oxide (GO) with electrospun scaffolds facilitated the cellular response. The greater mineralization was examined in GOcoated PC electrospun scaffolds (GPC) than PG scaffolds. The electrospun approach for the fabrication of PC and GPC scaffolds is presented in Fig. 1. The GO-coated PCL electrospun without CNCs is termed as PG scaffolds.

\section{Materials and methods}

\section{CNCs extraction from rice husk}

The laboratory cutting mill (IKA A10, IKA Labortechnik, Staufen, Germany) was used to achieve the fine powder of rice husks. Several steps were involved to obtain the CNCs from raw rice husks. Briefly, an alkaline treatment was completed to remove the silica content from the raw rice husks. For this, rice husk powder $(10 \mathrm{~g})$ was treated with $3 \%(\mathrm{w} / \mathrm{v})$ potassium hydroxide (KOH, Sigma-Aldrich) solution in a weight ratio of $1: 12$ at $85^{\circ} \mathrm{C}$ for $1 \mathrm{~h}$ followed by the filtration and washing with $10 \%(\mathrm{v} / \mathrm{v})$ hydrochloric acid ( $\mathrm{HCl}$, Wako Chemical) solution to achieve the neutral solution. The obtained material was repeatedly washed with distilled water and dried. After this, the obtained material was poured in distilled water in a ratio of $1: 60$ (solid to liquid) at $90^{\circ} \mathrm{C}$ for $30 \mathrm{~min}$ followed by the addition of sodium chlorite $\left(\mathrm{NaClO}_{2}\right.$, Daejung Chemical) and acetic acid (Wako Chemical) in the solution with continuous mechanical stirring for 6 times after $1 \mathrm{~h}$ time interval to remove the lignin byproduct. ${ }^{30,31}$ The obtained material was filtered and washed with distilled water and freeze-dried in a freeze dryer (FDU2200, EYELA, Tokyo, Japan) for $48 \mathrm{~h}$. The freeze-dried material was treated with $17.5 \%(\mathrm{w} / \mathrm{v})$ sodium hydroxide $(\mathrm{NaOH}$, SigmaAldrich) for $50 \mathrm{~min}$ followed by the addition of $10 \%(\mathrm{v} / \mathrm{v})$ acetic acid solution to remove the hemicellulose content from the material. The hydrolysis of cellulose was performed by using the $64 \%(\mathrm{w} / \mathrm{w})$ sulfuric acid $\left(\mathrm{H}_{2} \mathrm{SO}_{4}\right.$, Daejung Chemical) solution $45{ }^{\circ} \mathrm{C}$ for $1 \mathrm{~h}$. The obtained material was washed by using the dialysis tubing for 4 days. The photographs of the pure rice husk and CNCs suspension are presented in ESI Fig. 1. $\dagger$

\section{Preparation of GO and GO-coated substrates}

The GO was prepared by a modified Hummers' method. ${ }^{26,32}$ For this, the required amounts of graphite, powders were treated with $\mathrm{NaNO}_{3}$ in $\mathrm{H}_{2} \mathrm{SO}_{4}$ medium in an ice bath for $4 \mathrm{~h}$ followed by

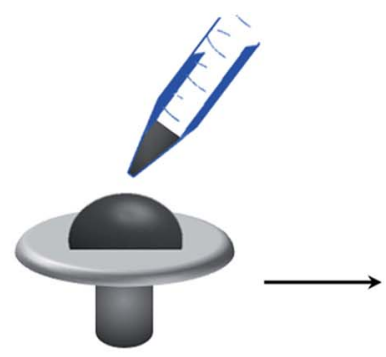

Graphene oxide (GO)

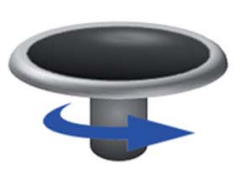

Spinning

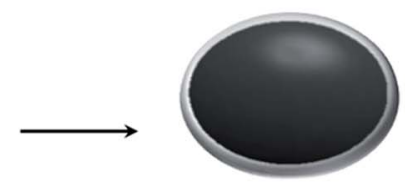

GO-coated substrates

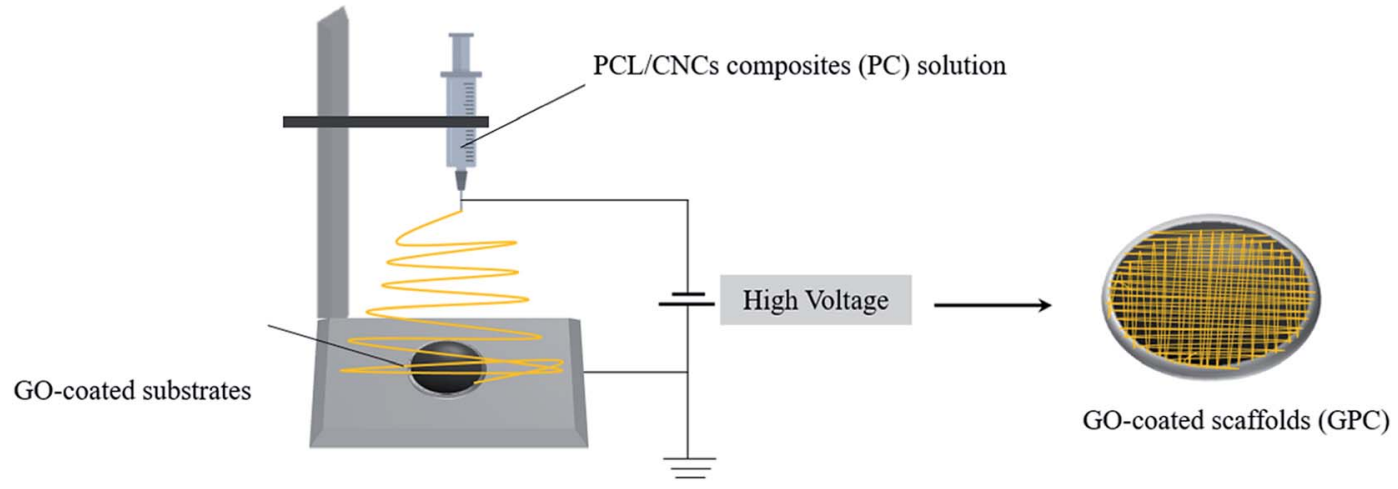

Fig. 1 Schematic representation of the formation of NPG scaffolds on graphene oxide-coated surface through electrospinning process. 
a.
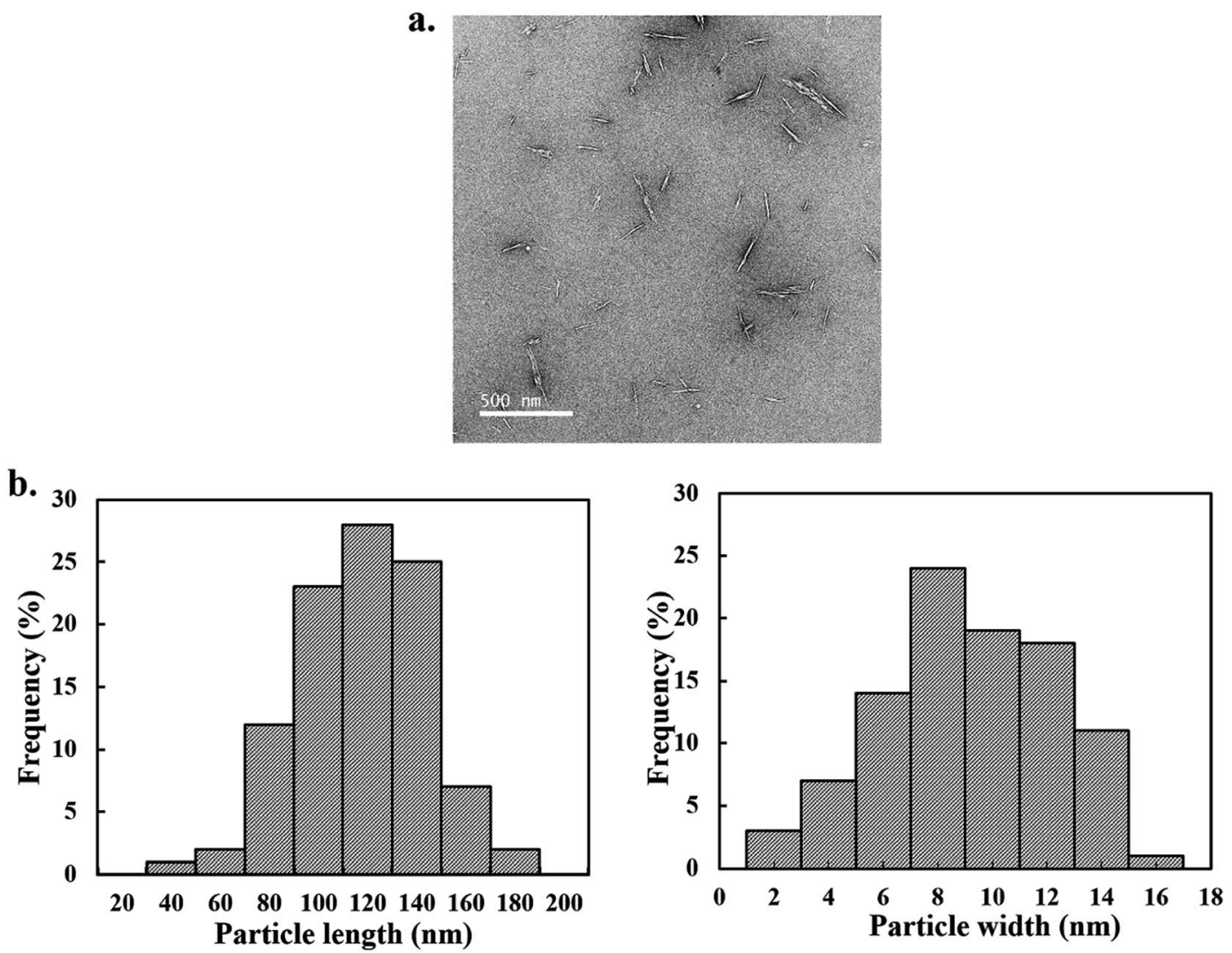

Fig. 2 Morphology of cellulose nanocrystals by TEM. (a) TEM images of CNCs, and (b) distribution histogram of length and width of acid hydrolyzed CNCs.

the addition of the $\mathrm{KMnO}_{4}$ powder and stirred this solution at $40{ }^{\circ} \mathrm{C}$ for $6 \mathrm{~h}$. After this, the suspension was heated in a water bath medium at $98{ }^{\circ} \mathrm{C}$ for $1 \mathrm{~h}$ followed by the addition of the $\mathrm{H}_{2} \mathrm{O}_{2}$ solution. The mixture was washed with $10 \% \mathrm{HCl}$ and deionized water several times followed by drying under the vacuum. The coating of glass substrates with GO was done as follows. First of all, glass substrates were cleaned by $70 \%$ ethanol, and then an addition of GO solution ( $70 \mu \mathrm{L}$ each) was added onto the glass substrates and immediately spun on a spin-coater as the conditions presented in ESI Table $1 . \dagger$ After coating, the GO-coated substrates were left to dry at room temperature for $24 \mathrm{~h}$.

\section{Characterization of CNCs}

Scanning electron microscopy (SEM) (Hitachi S-4800, Tokyo, Japan) was used for surface morphology observation of the pure rice husk and the chemically treated samples. The samples were utilized before the acid hydrolysis termed as the chemically treated sample. Atomic force microscopy (AFM) (Nanoscope 5, Bruker, Billerica, MA, USA) was used to monitor the morphology of rice husk derived CNCs. The thermal stability of rice huskderived materials was evaluated by the thermogravimetric analyzer (TGA) in the range of $40-500{ }^{\circ} \mathrm{C}$ with a heating rate of $10^{\circ} \mathrm{C} \mathrm{min}^{-1}$ (SDT Q600, TA Instruments, New Castle, USA). The shape and size distribution of CNCs was measured by transmission electron microscopy (TEM) (JEM 2100 F, Jeol, Tokyo,
Japan). For TEM, the sample was dispersed in deionized water by the ultra-sonication process. A drop of diluted CNCs suspension was deposited on the micro-grid and air-dried it for $45 \mathrm{~min}$. The CNCs dimensions and images were further analyzed using ImageJ software (version 1.8.0_112, Wayen Rasband, US National Institutes of Health, Bethesda, MD, USA; website: http:// www.imagej.nih.gov/ij/). The interactions between PCL and CNCs were monitored by Fourier-transform infrared spectroscopy (FTIR, PerkinElmer, Buckinghamshire, UK) in the scanning range of $500-4000 \mathrm{~cm}^{-1}$ at a resolution of $4 \mathrm{~cm}^{-1}$.

\section{Preparation of GPC scaffolds}

PCL $\left(M_{\mathrm{n}} \sim 80000\right.$, Sigma-Aldrich) was taken for the preparation of PC composites. For this, the required amount of PCL was dissolved in dimethylformamide (DMF) (Daejung Chemicals, Siheung, Republic of Korea) solvent to achieve the $15 \%(\mathrm{w} / \mathrm{v})$ solution. CNCs in different weight amounts $(0.1,0.5,1$, and $2 \%)$ were incorporated in a PCL solution with continuous mechanical stirring for $6 \mathrm{~h}$. A $10 \mathrm{~cm}^{3}$ plastic syringe was used for the electrospinning process to fabricate the fiber. The solution was delivered at a constant flow rate $\left(1 \mathrm{~mL} \mathrm{~h}^{-1}, 15 \mathrm{~cm}\right)$ to the GO-coated substrates connected to a high voltage power supply. On applying a high voltage $\left(18.0 \mathrm{kV} \mathrm{cm}^{-1}\right)$, a Taylor cone jet was ejected from the graphene-coated substrates. Prior to the cell seeding, the scaffolds were soaked in $70 \%$ ethanol solution for $24 \mathrm{~h}$ and washed thoroughly with deionized water. 
a.

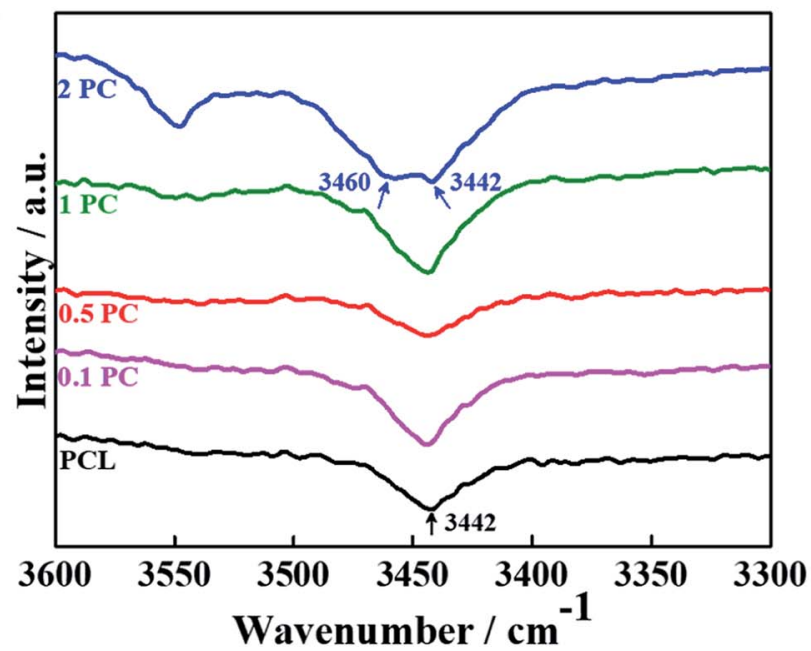

b.
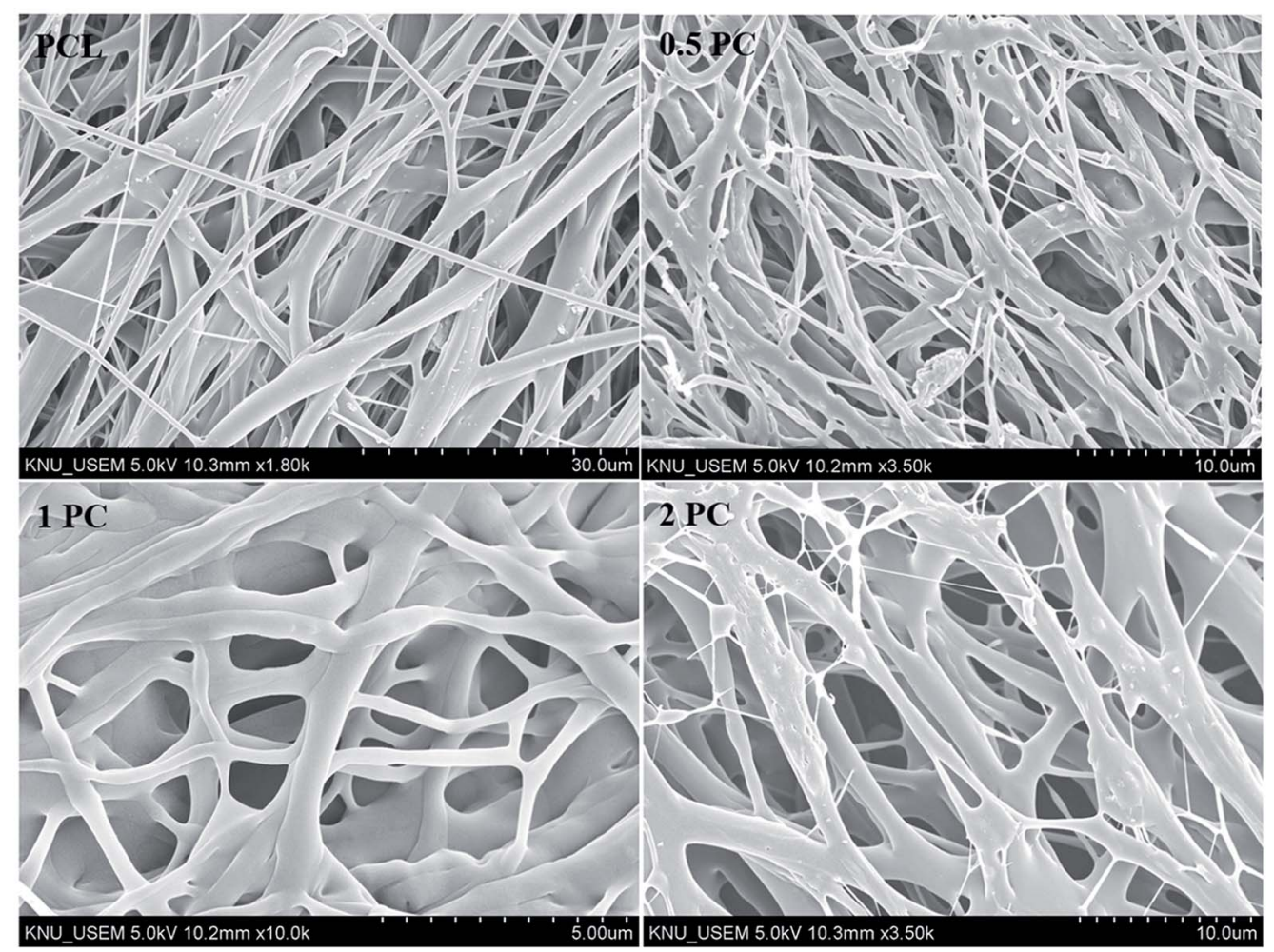

Fig. 3 (a) FTIR spectra of pure PCL and its indicated electrospun composite scaffolds, and (b) FE-SEM morphologies of pure PCL and its indicated electrospun composite scaffolds.

\section{Mechanical properties of PC scaffolds}

The mechanical strength of the scaffolds was evaluated through the tensile test. The tensile testing was carried out by using the universal testing machine (MCT-1150, AND Inc., Tokyo, Japan) (UTM). The analysis was performed at the elongation speed of $10 \mathrm{~mm} \mathrm{~min}^{-1}$ in triplicate manners.

\section{Cell culture}

The human mesenchymal stem cells (hMSCs) derived from the bone (Korean Cell Line Bank, Republic of Korea) were cultured in Dulbecco's Modified Eagle's Medium (DMEM) (Welgene, Seoul,
Republic of Korea) with $10 \%$ fetal bovine serum (Welgene, Seoul, Republic of Korea) and 1\% antibiotics (Welgene, Seoul, Republic of Korea). The cells were incubated at $37{ }^{\circ} \mathrm{C}$ in a humidified atmosphere with $5 \% \mathrm{CO}_{2}$ (Steri-Cycle 370 Incubator, Thermo Fischer Scientific, USA) environment. The old culture media were replaced with fresh media every 3 days' time intervals during the experiment. After $\sim 80-90 \%$ of cell confluency, the cells were detached with $1 \mathrm{~mL}$ trypsin-ethylene diamine tetra acetic acid (EDTA, Gibco, Waltham, Massachusetts, USA) solution followed by the rinsing with phosphate-buffered saline (PBS). The cells were used between three and five passages. 

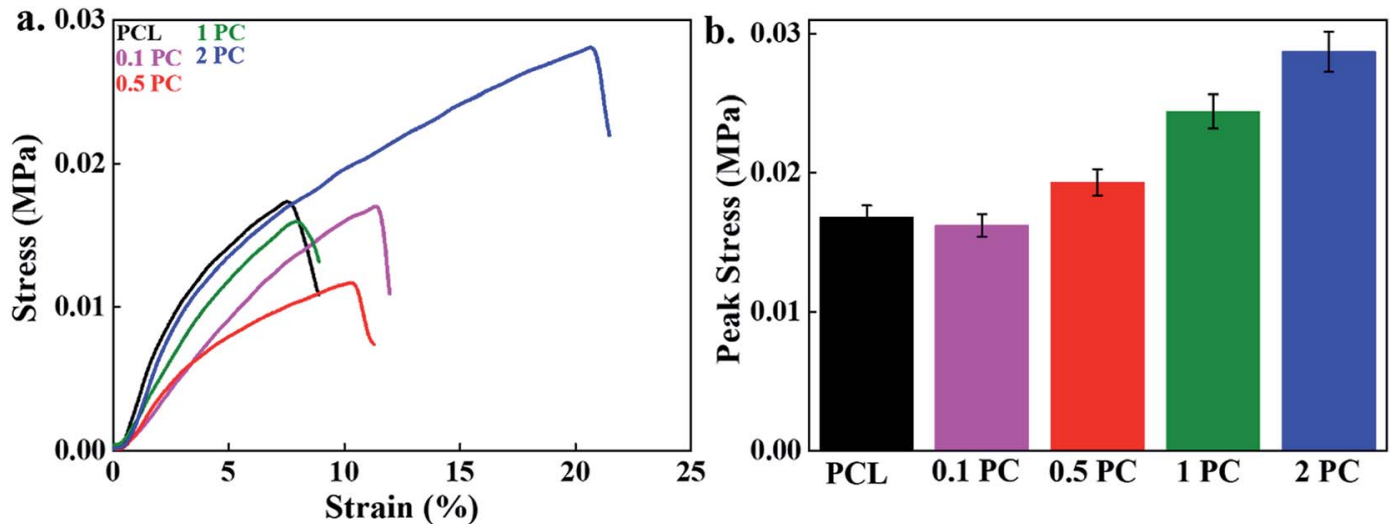

Fig. 4 The mechanical properties of the scaffolds; (a) the strain-stress curve, and (b) the bar diagram representation of peak stress values of different scaffolds under uniaxial tensile test.

The fabricated scaffolds were placed in a 24 -well plate and were incubated at $37{ }^{\circ} \mathrm{C}$ in $5 \% \mathrm{CO}_{2}$ for different time periods. Cytotoxicity of the fabricated PC and GPC scaffolds was evaluated through WST-1 assay. For this, the cultured cells were treated with $100 \mu \mathrm{L}$ of WST-1 reagent to produce the formazan dye which was quantified with the spectrometer (Infinite ${ }^{\circledR} \mathbf{M}$ Nano 200 Pro, TECAN, Switzerland) by taking the absorbance at $490 \mathrm{~nm}$. The samples were taken in the triplicate fashion, and data are given as mean ODs \pm standard deviations.

\section{Mineralization potential of CNCs and GPC scaffolds}

The mineralization potential of rice-husk derived CNCs and GPC scaffolds was evaluated through alizarin red staining (ARS, Sigma-Aldrich) technique in the presence of hMSCs after 7 and 14 days of treatment. For this, the cells were cultured in the osteogenic medium and the old medium was replaced with fresh medium after 2 days' time intervals. The passage 4 cells were used for this experiment. After the required time periods ( 7 and 14 days), the cells were fixed with $4 \%$ formalin solution for $15 \mathrm{~min}$ and followed by air-dried for $30 \mathrm{~min}$. The excess dye was removed from the medium by repeated washing it with deionized water. The residual dye was dissolved in $500 \mu \mathrm{L}$ of destaining solution (10\% cetyl pyridinium chloride and $10 \mathrm{mM}$ sodium phosphate). The absorbance was taken at $562 \mathrm{~nm}$ using the spectrometer (Infinite ${ }^{\circledR}$ M Nano 200 Pro; TECAN, Switzerland).

\section{Reverse transcription-polymerase chain reaction (RT-PCR) analysis}

The expression of osteogenic differentiation-specific genes in the presence of rice husk derived CNCs was evaluated through the RT-PCR technique after 7 and 14 days of treatment. For this, the cells were cultured in a $60 \times 15 \mathrm{~mm}$ culture dish with osteogenic induction media at $37^{\circ} \mathrm{C}$ in $5 \% \mathrm{CO}_{2}$ condition for desired time periods. The total RNA was extracted through TRIzol® reagent (Thermo Fischer, USA) as per manufacture's protocols. The purity and concentration of extracted RNA were determined by a spectrophotometer. The cDNA was produced from the extracted RNA in the presence of reverse transcriptase (Superscript II RTase,
Invitrogen, Gaithersburg, MD, USA). The mRNA expression was measured through RT-PCR using a Bio-Rad Real-Time PCR (CFX96 ${ }^{\mathrm{TM}}$, Bio-Rad, USA) with 43 cycles of denaturation for $15 \mathrm{~s}$ at $95{ }^{\circ} \mathrm{C}$ and $1 \mathrm{~min}$ amplification at $60{ }^{\circ} \mathrm{C}$. All the reactions were done in the triplicate fashion and normalized with housekeeping gene hypoxanthine-guanine phosphoribosyl transferase (HPRT). The cycle threshold values were calculated and equated for checking the gene expression levels in the control and treated groups. The specific primer sets osteopontin (OPN), alkaline phosphatases (ALP), integrin binding sialoprotein (IBSP), and runt-related transcription factor 2 (RUNX2) used in this experiment are given in ESI Table $2 . \dagger$

\section{Statistical analysis}

Statistical analysis was accomplished with SAS for Windows v8.2 (SAS Institute, NC, USA). Statistical significance between the treated groups and control was performed with ANOVA, Duncan's multiple range test, and Mann-Whitney Rank Sum tests. All the data are shown as mean \pm standard deviations.

\section{Results and discussion}

\section{Characterization of chemically treated rice husk and GO}

The AFM image of rice-husk derived CNCs is given in ESI Fig. 2a. $\dagger$ AFM image clearly indicates that the formation of nanostructured cellulose has occurred by acid hydrolysis. The average length and diameter of the obtained material are approximately $135 \mathrm{~nm}$ and $15 \mathrm{~nm}$, respectively, which is closely related to TEM data. Thermal stability is an essential parameter of the material that reflects their stability for various applications. Differential thermal analysis (DTA) thermograms of the pure rice husk and chemically treated sample are shown in ESI Fig. 2b. $\dagger$ Both samples showed similar kinds of derivative peaks in their degradation pattern, and it started from $250{ }^{\circ} \mathrm{C}$ and $370{ }^{\circ} \mathrm{C}$. It has been observed that the decrease in thermal stability was found in the chemically treated sample compared to the pure rice husk. ${ }^{33}$ However, an enhancement in the thermal stability was seen in the chemically treated sample below $300{ }^{\circ} \mathrm{C}$ than pure rice husk. This was attributed to the 

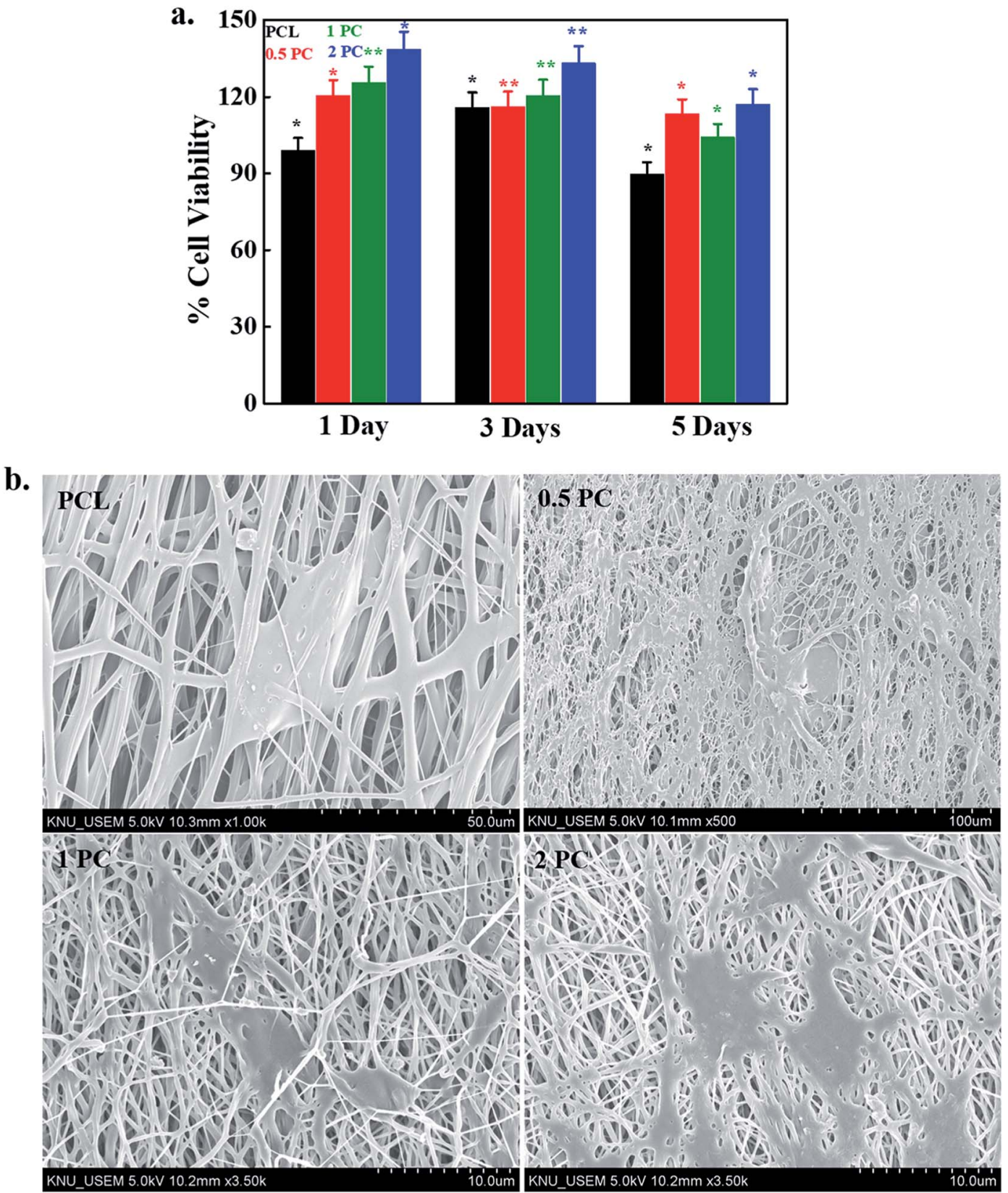

Fig. 5 Cytotoxicity evaluation of fabricated scaffolds; (a) cell viability data of pure PCL and its indicated electrospun composite scaffolds at indicated time intervals $\left(P^{*}<0.05\right)$, and (b) FE-SEM morphologies of hMSCs on the surface of pure PCL and its indicated electrospun composite scaffolds after 5 days of treatment.

presence of a compact cellulose structure that hinders the evaporation of volatile residues. ${ }^{34}$ Furthermore, the chemically treated sample $\left(>300^{\circ} \mathrm{C}\right)$ exhibited lower thermal stability than the pure rice husk. This was due to the presence of some lignin matrix in the pure rice husk that provides better thermal stability. ${ }^{35}$ The surface morphologies of the pure rice husk and the chemically treated samples are presented in ESI (Fig. 2c(i) and (ii) $\dagger$ ). The relatively smooth surface morphology was observed in the pure rice husk compared to the chemically treated due to the presence of cellulose layers in the form of microfibrils. Here, the sample used prior to the acid hydrolysis is termed as the chemically treated. The FTIR spectrum of the prepared GO is given in ESI Fig. $3 . \dagger$ The appearance of the characteristic absorption peaks in the FTIR spectrum clearly indicates the formation of GO has occurred.

\section{Morphology of CNCs}

The repeating units of the cellulose are linked by glycosidic bonds and it can be easily dissociated by a strong acid. The cleavage of glycosidic bonds is responsible for the formation of nanostructured cellulose. A TEM image of rice husk derived nanostructured cellulose after the acid hydrolysis is shown in Fig. 2a. The average length and width of the rice husk derived CNCs was 128.3 and $10.5 \mathrm{~nm}$, respectively. TEM image 


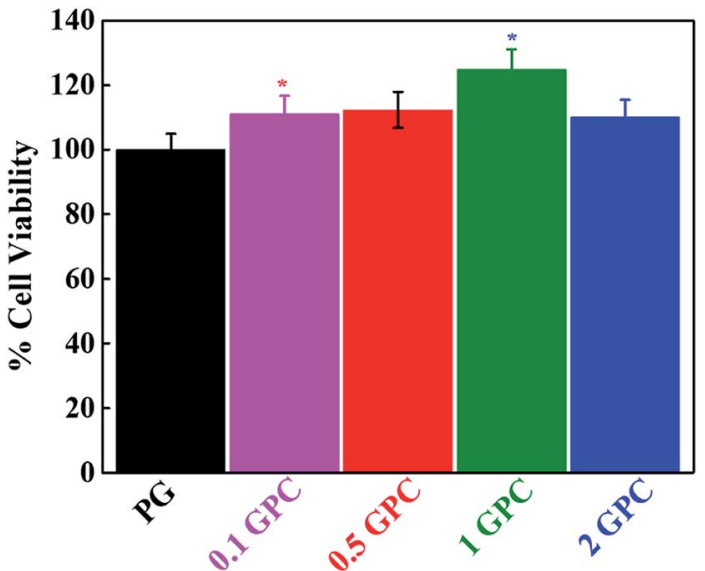

Fig. 6 Evaluation of cellular response of hMSCs in the presence of the fabricated scaffold after $24 \mathrm{~h}$ of treatment $\left(P^{*}<0.05\right)$.

indicated the generation of a highly ordered and crystalline structure had occurred through the acid hydrolysis by removing the amorphous zones from cellulose. Fig. 2b shows the distribution length and width of rice husk derived CNCs after the acid hydrolysis. The average length and the width of rice husk derived CNCs are similar to the earlier reported values. ${ }^{36}$

\section{Interactions and morphology of PCL/CNCs electrospun}

The interactions between the polymer chains and CNCs were revealed by FTIR spectroscopy and data are shown in Fig. 3a. The appearance of the absorption peak at $3442 \mathrm{~cm}^{-1}$ in FTIR spectra indicated the presence of hydroxyl groups $(-\mathrm{OH})$ in PCL and its indicated composite scaffolds. ${ }^{37}$ It was interesting to see that as the content of CNCs increased in the polymer matrix an appearance of another hump was observed in the higher frequency region $\left(3460 \mathrm{~cm}^{-1}\right.$ ) suggested the presence of free hydrogen-bonded hydroxyl groups. The appearance of the peak at higher region indicated that the interaction in-between polymer chains was decreased and interactions between polymer chains with CNCs were increased. The FTIR spectra of the pure PCL and its designated electrospun composite scaffolds in lower regions are given in ESI Fig. 4.† No significant changes were noted in the lower regions of the FTIR spectra of pure polymer and it's indicated composite scaffolds. This is due to the low concentration and overlapping CNCs peaks with pure polymer. ${ }^{38}$

The FE-SEM micrographs of the pure PCL polymer and its indicated composite scaffolds are given in Fig. 3b. The surface morphologies of the fabricated are affected by several factors such as nature of the solvent, concentration, and viscosity of the
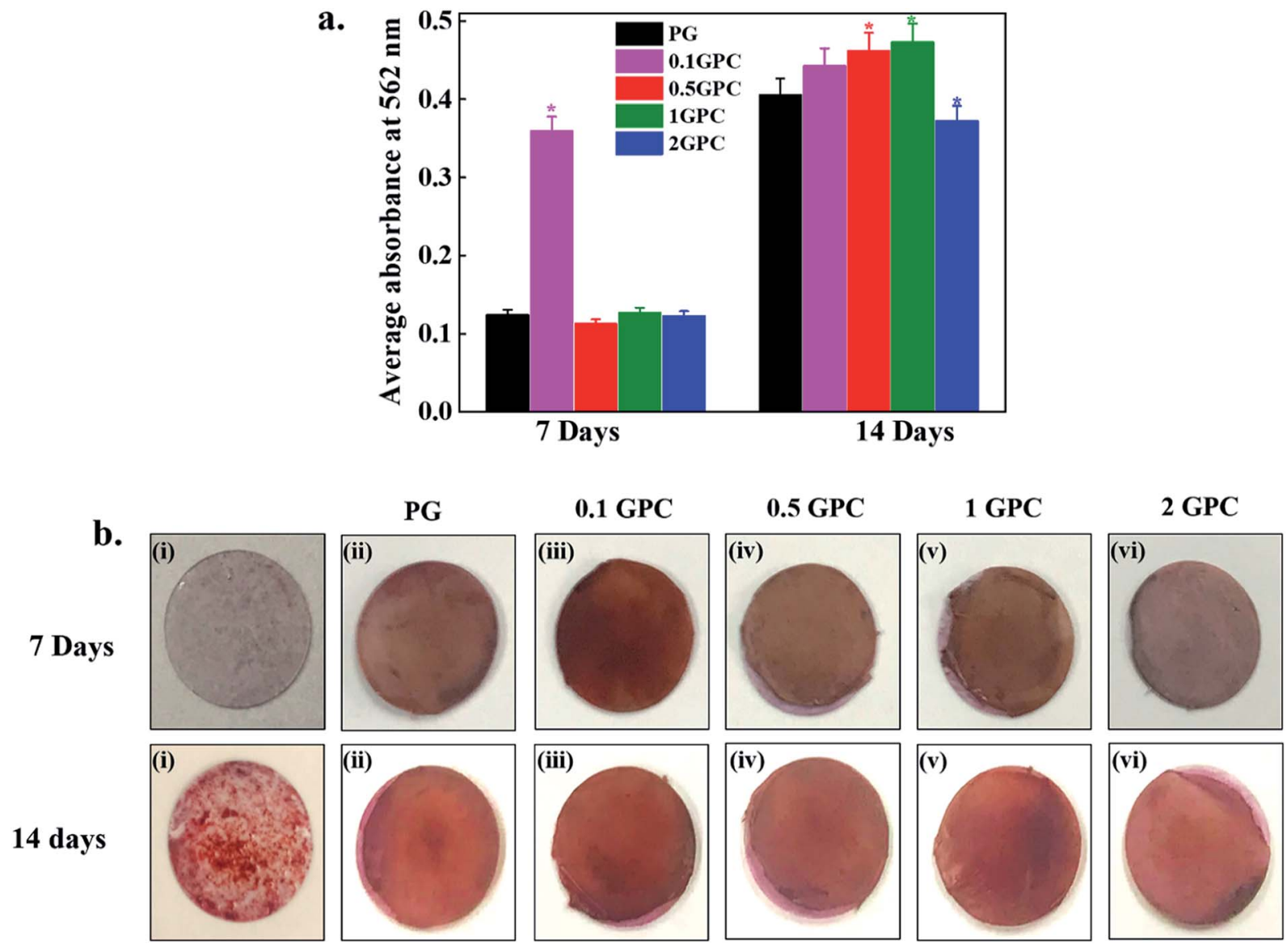

Fig. 7 Evaluation of the mineralization potential of GPC scaffolds at indicated time intervals; (a) quantitative analysis of the mineralization potential of GPC scaffolds ( $P^{*}<0.05$ ), and (b) alizarin staining of hMSCs cultured for 7 and 14 days on scaffolds; (i) control (ii) PG (iii) 0.1 (iv) 0.5 (v) 1 and (vi) 2 GPC scaffolds. 
solution, distance, charge density and applied voltage. ${ }^{\mathbf{1 8 - 2 0}}$ The fabricated scaffolds have smooth morphology without noticeable beads and thinner diameter was observed in PC scaffolds as compared to pure PCL scaffolds. A similar result was also observed by Shi et al. in the presence of CNCs with the poly(lactic acid) polymer matrix. ${ }^{39}$ Since CNCs have negatively charged sulfate groups in their structure which enhances the electrical conductivity of the electrospinning solution and consequently a decrease in viscosity has occurred. This decrease in the viscosity of electrospinning solution causes the formation of thinner diameter in PC scaffolds than pure polymer. Therefore, at a higher concentration of CNCs, the viscosity of the polymer solution gets decreases which leads to a decrease in the diameter of the scaffolds.

\section{Mechanical properties of PC scaffolds}

The mechanical strength of the fabricated (PC) scaffolds was evaluated by using the UTM, and results are given in Fig. 4a. The pure PCL polymer scaffold is treated as the control. A significant improvement in the elongation at break was observed in the fabricated scaffolds compared to the control. This was due to the filler-polymer interaction, stress transfer between polymer and/or filler and nucleation effect of nanomaterials on the crystallization of polymer. ${ }^{40}$ These reduced the crack propagation process during the experiment by the orientation of CNCs towards the applied force. An enhancement in the mechanical strength of PCL electrospun was also reported by Augustine et al. and this improvement in the mechanical strength was profoundly affected by the content of nanomaterial in the polymer matrix. A decrease in mechanical strength was noted at a higher content due to the agglomeration of nanomaterial. ${ }^{20,22,23,40}$ The elongation at break was 7.5, 11.5, 11.1, 13.6 , and $20.6 \%$ for the control, $0.1 \%, 0.5 \%, 1 \%$ and $2 \%$ incorporated $\mathrm{CNCs}$, respectively indicating the more flexible nature of the fabricated scaffolds than control. The tensile peak stress of the fabricated scaffolds is given in Fig. $4 \mathrm{~b}$. The stress at break of pure PCL scaffolds was found to be $0.0168 \mathrm{MPa}$. The stress at break of PC scaffolds at the $0.1 \%$ CNCs contents $(0.0162 \mathrm{MPa})$ was similar to the polymer scaffolds. However, it is interesting to note that enhancement in the stress at break of the fabricated scaffolds has occurred by increasing the content of the CNCs. The value of stress at break of PC scaffolds at 0.5, 1, and $2 \%$ was $0.0194,0.0244,0.0288 \mathrm{MPa}$, respectively. This was attributed to the effective stress transfer from polymer chains to CNCs during the experiment. The higher aspect ratio of CNCs enables a better platform for interactions and consequently improved the overall mechanical properties. The improvement in the thermal and mechanical strength was also reported in maleic anhydride (MAH) grafted poly(lactic acid) (PLA)/CNCs electrospun composite scaffolds. ${ }^{\mathbf{4 1}}$

\section{Cell viability and adhesion}

Cytotoxicity of the fabricated electrospun scaffolds was evaluated through MTT assay after different time intervals and given in Fig. 5a. The well without any sample was treated as a control. Notably better cell viability was observed in the presence of PC electrospun scaffolds than the pure polymer scaffold. An enhancement in the cell viability of PCL electrospun in the presence of nanofiller has been reported earlier for tissue engineering. ${ }^{16-24}$ The higher cell viability on composite scaffolds compared to pure PCL scaffold demonstrates the more biocompatible nature of PC scaffolds. Cell adhesion efficiency indicates the possible application of the scaffolds as a transplant material. It also suggests how the cells grow and adhered onto a fabricated material. ${ }^{42}$ Cellular responses like cell adhesion, proliferation, and differentiations are extremely influenced by the surface modification and charge, size, structural defect and surface wettability. ${ }^{43}$ Cell adhesion potential of hMSCs on the surface of pure PCL and its indicated composite scaffolds after 5 days of treatment was demonstrated by SEM images and morphologies are shown in Fig. 5b. These results indicated that the cells were properly adhered on the surface of the fabricated scaffolds by filopodia like structures. It was interesting to note that the composite scaffolds have a higher cell population than the pure polymer suggested their improve biocompatibility.

\section{Cytotoxicity of GPC scaffolds}

For tissue engineering applications, the material should have some specific properties like non-toxicity and biodegradability. Cytotoxicity of the fabricated GPC scaffolds was monitored by WST-1 assay in the presence of hMSCs after $24 \mathrm{~h}$ of treatment and shown in Fig. 6. Notably, better cell viability was observed on the surface of GO-coated (GPC) scaffolds compared to the control (PG) and this finding was profoundly affected by the content of CNCs. It was noted that the cell viability was increased by increasing the content of CNCs in the scaffolds. However, this enhancement was further decreased after a certain concentration of CNCs $(1 \%)$ indicated that $1 \%$ CNCs is an optimum concentration for scaffold fabrication. This result indicated that the fabricated scaffolds were biocompatible in nature. The higher surface area and the porosity of the fabricated scaffolds provide better conditions for enhanced cellular activity. ${ }^{44}$

\section{Mineralization efficiency of GPC scaffolds}

The efficiency of mineral deposition indicates osteogenesis and has been treated as a bone regeneration marker. The mineralization nodule formation potential of CNCs after 7 and 14 days of treatment is given in ESI Fig. 5. $\uparrow$ The mineralized test was performed with the alizarin red solution (ARS). This result clearly indicates that the 1\% CNCs have higher mineralization ability than others and the control. It is wellknown that the osteogenic process is highly affected by the structure, as well as the physiochemical properties of the fabricated scaffolds. ${ }^{45}$ Therefore, polymer nanofiber with bioactive materials can further enhance the biomimetic potentials of the scaffolds and facilitate cell attachment, proliferation, and differentiation. ${ }^{46}$ The ARS was performed to evaluate the effect of the GPC scaffolds on hMSCs differentiation after 7 and 14 days of treatment. It was observed that the scaffolds with $0.1 \%$ CNCs exhibited a higher ability 
of cell differentiation after 7 days of treatment. Moreover, an enhancement in mineralization was observed with increasing CNCs content after 14 days of treatment. This indicated that $0.1 \%$ CNC was effective for the mineralization during the early stage of osteogenic differentiation and 1\% CNC was most effective at the later stage of differentiation. This is due to the better cellular response from the scaffolds having $1 \%$ CNC in the polymer matrix. This kind of trend is reported earlier for alkaline phosphatase activity (ALP) an early osteogenic differentiation marker in GO incorporated poly(lactide-co-glycolide)/hydroxyapatite

(GO-PLGA/HA) microcarriers. ${ }^{47}$ The quantitative analysis for mineralization potential of GPC scaffolds is presented in Fig. 7a. It was interesting to see that this enhancement was also affected by CNCs contents and after a certain (1\%) concentration decrease in the mineralization has occurred suggested that $1 \% \mathrm{CNC}$ is the optimum concentration for mineralization. The PCR analysis for the expression of osteogenic differentiation-specific genes at $1 \%$ CNCs concentration is given in ESI Fig. $6 . \dagger$ The expression of osteogenic gene markers in the presence of CNCs clearly indicates their osteogenic potential. The visual images of stained GPC scaffolds after 7 and 14 days of treatment are shown in Fig. $7 \mathrm{~b}$. The more intense color was observed in $0.1 \%$ GPC scaffolds compared to the control and other scaffolds after 7 days of treatment. However, the more intense image was noted in $1 \%$ GPC scaffolds after the 14 days of incubation further confirm our observation. CNCs can be used as an implant material for tissue engineering applications. ${ }^{48,49}$ These results indicate that CNCs-based materials can promote cell proliferation, spreading, and differentiation. Therefore, the developed scaffolds have the potential to use as a biomaterial for tissue engineering, especially bone tissues.

\section{Conclusions}

CNCs were successfully isolated from rice husks biomass through the acid hydrolysis for tissue engineering. CNCs were characterized through TEM and AFM. The PC scaffolds were fabricated through the electrospinning technique by varying CNCs content in the PCL matrix. An improvement in the mechanical strength was noted in PC composite scaffolds compared to the pure polymer scaffolds due to the better interfacial interactions. No significant cytotoxicity was observed in the presence of PC scaffolds and cells were properly adhered to the surface of PC scaffolds. Furthermore, an enhancement in the cellular response was noted in GO-coated PC scaffolds indicated their improved biocompatibility. Additionally, higher mineralization was noted with GO-coated PC (GPC) scaffolds vis-à-vis PG scaffolds indicated their greater mineralization potential. Notably better biocompatibility along with higher osteogenic potential makes GPC scaffolds a suitable biomaterial for tissue engineering applications. We anticipate that this approach for the fabrication of PCL-based scaffolds will provide a new pathway in the field of tissue engineering for bone regeneration.

\section{Conflicts of interest}

The authors declare no competing financial interests.

\section{Abbreviations}

CNCs Cellulose nanocrystals

PC Poly( $\varepsilon$-caprolactone)/cellulose nanocrystals

GPC GO-coated poly( $\varepsilon$-caprolactone)/cellulose nanocrystals

PG GO-coated poly( $\varepsilon$-caprolactone)

\section{Acknowledgements}

This research was supported by Basic Science Research Program through the National Research Foundation of Korea (NRF) funded by the Ministry of Education (No. 2018R1A6A1A03025582, No. 2019R1D1A3A03103828). The authors also thankful to the Cooperative Research Program for Agricultural Science and Technology Development (No. PJ012854012017), Rural Development Administration, Republic of Korea.

\section{References}

1 B. Dhandayuthapani, Y. Yoshida, T. Maekawa and D. Kumar, Int. J. Polym. Sci., 2011, 2011, 1-19.

2 D. Hutmacher, Biomaterials, 2000, 21, 2529-2543.

3 S. Agarwal, J. Wendorff and A. Greiner, Polymer, 2008, 49, 5603-5621.

4 Q. Pham, U. Sharma and A. Mikos, Tissue Eng., 2006, 12, 1197-1211.

5 Q. Shi, C. Zhou, Y. Yue, W. Guo, Y. Wu and Q. Wu, Carbohydr. Polym., 2012, 90, 301-308.

6 Z. Huang, Y. Zhang, M. Kotaki and S. Ramakrishna, Compos. Sci. Technol., 2003, 63, 2223-2253.

7 N. Mei, G. Chen, P. Zhou, X. Chen, Z. Shao, L. Pan and C. Wu, J. Biomater. Appl., 2005, 19, 323-339.

8 S. Cho, S. Jung, M. Kang, H. Shin and J. Youk, Polymer, 2015, 69, 95-102.

9 J. George, M. Sreekala and S. Thomas, Polym. Eng. Sci., 2001, 41, 1471-1485.

10 C. Salas, T. Nypelö, C. Rodriguez-Abreu, C. Carrillo and O. Rojas, Curr. Opin. Colloid Interface Sci., 2014, 19, 383-396.

11 R. Domingues, S. Chiera, P. Gershovich, A. Motta, R. Reis and M. Gomes, Adv. Healthcare Mater., 2016, 5, 1364-1375.

12 Y. Habibi, L. Lucia and O. Rojas, Chem. Rev., 2010, 110, 3479-3500.

13 Y. R. Seo, J. W. Kim, S. Hoon, J. Kim, J. H. Chung and K. T. Lim, J. Biosyst. Eng., 2018, 43, 59-71.

14 A. Ghanbari, T. Tabarsa, A. Ashori, A. Shakeri and M. Mashkour, Carbohydr. Polym., 2018, 197, 305-311.

15 A. Sinha, E. Martin, K. Lim, D. Carrier, H. Han, V. Zharov and J. Kim, J. Biosyst. Eng., 2015, 40, 373-393.

16 R. Augustine, E. Dominic, I. Reju, B. Kaimal, N. Kalarikkal and S. Thomas, RSC Adv., 2014, 4, 24777-24785. 
17 R. Augustine, E. Dominic, I. Reju, B. Kaimal, N. Kalarikkal and S. Thomas, RSC Adv., 2014, 4, 51528-51536.

18 R. Augustine, E. Dominic, I. Reju, B. Kaimal, N. Kalarikkal and S. Thomas, J. Biomed. Mater. Res., Part B, 2014, 103, 1445-1454.

19 B. Joseph, R. Augustine, N. Kalarikkal, S. Thomas, B. Seantier and Y. Grohens, Mater. Today Commun., 2019, 19, 319-335.

20 R. Augustine, S. Nethi, N. Kalarikkal, S. Thomas and C. Patra, J. Mater. Chem. B, 2017, 5, 4660-4672.

21 R. Augustine, A. Saha, V. Jayachandran, S. Thomas and N. Kalarikkal, Int. J. Polym. Mater., 2015, 64, 526-533.

22 S. Nandagopal, R. Augustine, S. George, V. P. Jayachandran, N. Kalarikkal and S. Thomas, Polym.-Plast. Technol. Eng., 2016, 55, 1785-1796.

23 R. Augustine, Y. Dalvi, V. Yadu Nath, R. Varghese, V. Raghuveeran, A. Hasan, S. Thomas and N. Sandhyarani, Mater. Sci. Eng., C, 2019, 103, 109801-109814.

24 R. Augustine, A. Hasan, N. Patan, A. Augustine, Y. Dalvi, R. Varghese, R. Unni, N. Kalarikkal, A. Al Moustafa and S. Thomas, Macromol. Biosci., 2019, 19, 1900058-1900073.

25 C. Ariza, A. Fleury, C. Tormos, V. Petruk, S. Chawla, J. Oh, D. Sakaguchi and S. Mallapragada, Stem Cell Rev. Rep., 2010, 6, 585-600.

26 K. Lim, H. Seonwoo, K. Choi, H. Jin, K. Jang, J. Kim, J. Kim, S. Kim, P. Choung and J. Chung, Adv. Healthcare Mater., 2016, 5, 2069-2079.

27 J. Unagolla and A. Jayasuriya, Mater. Sci. Eng., C, 2019, 102, 1-11.

28 M. Turk and A. Deliormanlı, J. Biomater. Appl., 2017, 32, 2839.

29 K. Hu, D. Kulkarni, I. Choi and V. Tsukruk, Prog. Polym. Sci., 2014, 39, 1934-1972.

30 L. Ludueña, D. Fasce, V. A. Alvarez and P. M. Stefani, BioResources, 2011, 6, 1440-1453.

31 A. Daifullah, N. Awwad and S. El-Reefy, Chem. Eng. Process., 2004, 43, 193-201.
32 Y. Lee, W. Jang, H. Im and J. Sung, Curr. Appl. Phys., 2015, 15, S95-S102.

33 M. Roman and W. Winter, Biomacromolecules, 2004, 5, 16711677.

34 H. Zhang, Y. Xu, Y. Li, Z. Lu, S. Cao, M. Fan, L. Huang and L. Chen, Polymers, 2017, 9, 526-528.

35 H. Mi, X. Jing, J. Peng, M. Salick, X. Peng and L. Turng, Cellulose, 2014, 21, 2727-2741.

36 N. Johar and I. Ahmad, BioResources, 2012, 7, 5469-5477.

37 A. Benkaddour, K. Jradi, S. Robert and C. Daneault, Nanomaterials, 2013, 3, 141-157.

38 A. Hivechi, S. Bahrami and R. Siegel, Mater. Sci. Eng., C, 2019, 94, 929-937.

39 Q. Shi, C. Zhou, Y. Yue, W. Guo, Y. Wu and Q. Wu, Carbohydr. Polym., 2012, 90, 301-308.

40 R. Augustine, H. Malik, D. Singhal, A. Mukherjee, D. Malakar, N. Kalarikkal and S. Thomas, J. Polym. Res., 2014, 21, 347-364.

41 C. Zhou, Q. Shi, W. Guo, L. Terrell, A. Qureshi, D. Hayes and Q. Wu, ACS Appl. Mater. Interfaces, 2013, 5, 3847-3854.

42 D. Patel, V. Gupta, A. Dwivedi, S. Pandey, V. Aswal, D. Rana and P. Maiti, Polymer, 2016, 106, 109-119.

43 K. Yang, Y. Li, X. Tan, R. Peng and Z. Liu, Small, 2012, 9, 1492-1503.

44 H. Yoshimoto, Y. Shin, H. Terai and J. Vacanti, Biomaterials, 2003, 24, 2077-2082.

45 S. Kwak, A. Haider, K. Gupta, S. Kim and I. Kang, Nanoscale Res. Lett., 2016, 11, 323-339.

46 M. Frohbergh, A. Katsman, G. Botta, P. Lazarovici, C. Schauer, U. Wegst and P. Lelkes, Biomaterials, 2012, 33, 9167-9178.

47 C. Fu, X. Yang, S. Tan and L. Song, Sci. Rep., 2017, 7, 1254912562.

48 Q. Chen, R. Garcia, J. Munoz, U. Pérez de Larraya, N. Garmendia, Q. Yao and A. Boccaccini, ACS Appl. Mater. Interfaces, 2015, 7, 24715-24725.

49 H. Jafari, M. Shahrousvand and B. Kaffashi, ACS Biomater. Sci. Eng., 2018, 4, 2484-2493. 